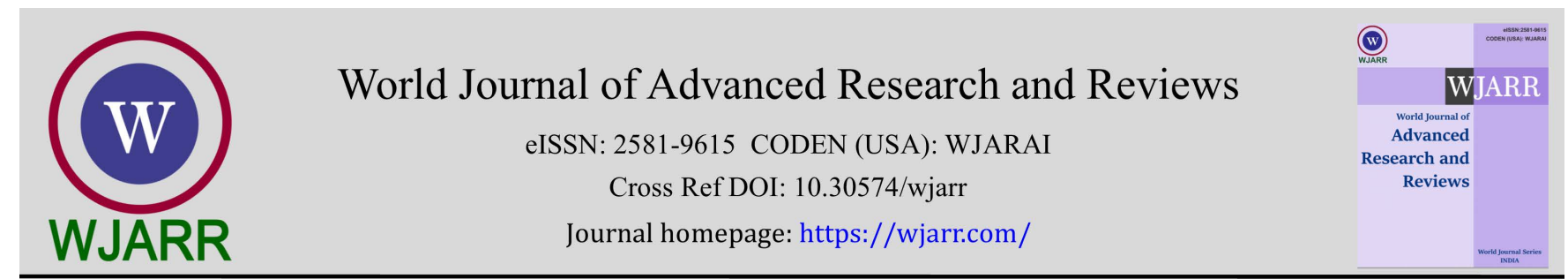

(REVIEW ARTICLE)

\title{
On class (n, mBQ) Operators
}

\author{
Wanjala Victor * and Beatrice Adhiambo Obiero \\ Department of Mathematics and computing, Rongo University, Kitere Hills Kenya.
}

World Journal of Advanced Research and Reviews, 2021, 11(02), 053-057

Publication history: Received on 26 June 2021; revised on 02 August 2021; accepted on 05 August 2021

Article DOI: https://doi.org/10.30574/wjarr.2021.11.2.0356

\begin{abstract}
In this paper, we introduce the class of $(n, m B Q)$ operators acting on a complex Hilbert space $H$. An operator if $T \in B(H)$ is said to belong to class ( $\mathrm{n}, \mathrm{mBQ})$ if $\mathrm{T} * 2 \mathrm{~m} \mathrm{~T}{ }^{2 \mathrm{n}}$ commutes with ( $\left.\mathrm{T} * \mathrm{~m} \mathrm{~T}^{\mathrm{n}}\right)^{2}$ equivalently [T $\left.* 2 \mathrm{mT} 2 \mathrm{n},\left(\mathrm{T} * \mathrm{mT}^{\mathrm{n}}\right)^{2}\right]=0$, for a positive integers $n$ and $m$. We investigate algebraic properties that this class enjoys. Have. We analyze the relation of this class to $(\mathrm{n}, \mathrm{m})$-power class $(\mathrm{Q})$ operators.
\end{abstract}

Keywords: (n,m)-power Class (Q); Normal; Binormal operators; N-power class (Q); (BQ) operators; (n,mBQ) operators

\section{Introduction}

$\mathrm{H}$ denotes Hilbert space over the complex field throughout this paper while $\mathrm{B}(\mathrm{H})$ the Banach algebra of all bounded linear algebra on an infinite dimensional separable Hilbert space $\mathrm{H}$. A bounded linear operator $\mathrm{T}$ is said to be in class (Q) if $\mathrm{T}^{* 2} \mathrm{~T}^{2}=(\mathrm{T} * \mathrm{~T})^{2}(2),(\mathrm{n}, \mathrm{m})$-power class $(\mathrm{Q})$ if $\mathrm{T} * 2 \mathrm{~m} \mathrm{~T}^{2 \mathrm{n}}=\left(\mathrm{T} * \mathrm{~m} \mathrm{~T}^{\mathrm{n}}\right)^{2}$ for positive integers $\mathrm{n}$ and $\mathrm{m}(1)$.

The class of (Q) operators was expanded to many classes such as the following classes, almost class (Q) (4), n-power class (Q) (2), $(\alpha, \beta)$-class (Q) (3), K* Quasi-n- Class (Q) Operators (6) and quasi M class (Q). An operator T $\in B(H)$ is said to belong to class (BQ) if $\mathrm{T}^{* 2} \mathrm{~T}^{2}(\mathrm{~T} * \mathrm{~T})^{2}=(\mathrm{T} * \mathrm{~T}){ }^{2} \mathrm{~T}{ }^{* 2} \mathrm{~T}^{2}(5), \mathrm{T} \in \mathrm{B}(\mathrm{H})$ is said to belong to class $(\mathrm{n}, \mathrm{mBQ})$ if $\mathrm{T} * 2 \mathrm{~m} T{ }^{2 \mathrm{n}}(\mathrm{T}$ $\left.{ }^{* m} \mathrm{~T}^{\mathrm{n}}\right)^{2}=\left(\mathrm{T} * \mathrm{~m} \mathrm{~T}^{\mathrm{n}}\right)^{2} \mathrm{~T}{ }^{* 2 \mathrm{~m}} \mathrm{~T}^{2 \mathrm{n}}$. A conjugation on a Hilbert space $\mathrm{H}$ is an anti-linear operator $\mathrm{C}$ from Hilbert space $\mathrm{H}$ onto itself that satisfies. $\mathrm{C} \xi, \mathrm{C} \zeta \mathrm{i}=\mathrm{h} \zeta, \xi \mathrm{i}$ for every $\xi, \zeta \in \mathrm{H}$ and $\mathrm{C}^{2}=\mathrm{I}$. An operator $\mathrm{T}$ is said to be complex symmetric if $\mathrm{T}=\mathrm{CT}{ }^{*} \mathrm{C}$.

\section{Main results}

\subsection{Theorem 1}

Let $T \in B(H)$ be such that $T \in(n, m B Q)$, then the following holds for $(N, m B Q)$;

(i). $\lambda \mathrm{T}$ for any real $\lambda$

(ii). Any $\mathrm{S} \in \mathrm{B}(\mathrm{H})$ that is unitarily equivalent to $\mathrm{T}$.

(iii). the restriction $\mathrm{T} / \mathrm{M}$ to any closed subspace $\mathrm{M}$ of $\mathrm{H}$.

Proof. (i). the proof is straight forward.

(ii). Let $\mathrm{S} \in \mathrm{B}(\mathrm{H})$ be unitarily equivalent to $\mathrm{T}$, then there exists a unitary operator $\mathrm{U}$

\footnotetext{
* Corresponding author: Wanjala Victor

Department of Mathematics and computing, Rongo University, Kitere Hills Kenya.

Copyright $(2021$ Author(s) retain the copyright of this article. This article is published under the terms of the Creative Commons Attribution Liscense 4.0.
} 
$\in B(H)$ with

$S^{n}=U{ }^{*} T{ }^{n} U$ and $S^{* m}=U{ }^{*} T * m$ for non-negative integers $n$ and $m$. Since $T \in(n, m B Q)$, we have;

$\mathrm{T} * 2 \mathrm{~m} \mathrm{~T}^{2 \mathrm{n}}\left(\mathrm{T} * \mathrm{~m} \mathrm{~T}^{\mathrm{n}}\right)^{2}=\left(\mathrm{T} * \mathrm{~m} \mathrm{~T}^{\mathrm{n}}\right)^{2} \mathrm{~T}^{* 2 \mathrm{~m}} \mathrm{~T}^{2 \mathrm{n}}$, hence

$\mathrm{S}^{* 2 \mathrm{~m}} \mathrm{~S}^{2 \mathrm{n}}\left(\mathrm{S}^{* \mathrm{~m}} \mathrm{~S}^{\mathrm{n}}\right)^{2}=\mathrm{UT} * 2 \mathrm{~m} U * \mathrm{UT}{ }^{2 \mathrm{n}} \mathrm{U} *\left(\mathrm{UT} * \mathrm{~m} \mathrm{U} * \mathrm{UT} \mathrm{n}^{\mathrm{U}}\right)^{2}$

$=\mathrm{UT} * 2 \mathrm{~m} U * \mathrm{U} * \mathrm{~T}{ }^{2 \mathrm{n}} \mathrm{U} * \mathrm{UT} * \mathrm{~m} U$ *UT *mU *UT n $\mathrm{U} * \mathrm{UT}$ nU *

$=\mathrm{UT} * 2 \mathrm{~m} \mathrm{~T}^{2 \mathrm{n}}\left(\mathrm{T} * \mathrm{~m} \mathrm{Tn}^{2}\right)^{2} \mathrm{U} *$

$=\mathrm{U}\left(\mathrm{T} * \mathrm{~m}^{\mathrm{n}}\right)^{2} \mathrm{~T}^{* 2 \mathrm{~m} T} \mathrm{~T}^{2 \mathrm{n}} \mathrm{U} *$

And

$\left(\mathrm{S}^{* \mathrm{~m}} \mathrm{~S}^{\mathrm{n}}\right)^{2} \mathrm{~S}^{* 2 \mathrm{~m}} \mathrm{~S}^{2 \mathrm{n}}=\left(\mathrm{UT} * \mathrm{mU} * \mathrm{UT} \mathrm{nU}^{*}\right)^{2} \mathrm{UT} * 2 \mathrm{mU} * \mathrm{UT} 2 \mathrm{nU} *$

$=\mathrm{UT} * \mathrm{~m} U * \mathrm{UT}{ }^{\mathrm{n}} \mathrm{U} * \mathrm{UT} * \mathrm{~m} \mathrm{U} * \mathrm{UT}{ }^{\mathrm{n} U}{ }^{*} \mathrm{UT} * 2 \mathrm{~m} U * \mathrm{UT}{ }^{2 \mathrm{n}} \mathrm{U} *$

$=\mathrm{UT} * \mathrm{~m} \mathrm{~T} \mathrm{n} \mathrm{T} * \mathrm{~m} \mathrm{~T}{ }^{n} \mathrm{~T} * 2 \mathrm{~m} \mathrm{~T} * 2 \mathrm{~m} U *$

$=U\left(T * m T^{n}\right)^{2} T^{* 2 m} T^{2 n} U^{*}$

Thus $\mathrm{S}$ is unitarily equivalent to $\mathrm{T}$.

(iii) . If $T$ is in class ( $n, m B Q)$, then;

$\mathrm{T}^{* 2 \mathrm{~m}} \mathrm{~T}^{2 \mathrm{n}}\left(\mathrm{T}^{* \mathrm{~m}} \mathrm{~T}^{\mathrm{n}}\right)^{2}=\left(\mathrm{T} * \mathrm{~m} \mathrm{~T}^{\mathrm{n}}\right)^{2} \mathrm{~T}^{* 2 \mathrm{~m}} \mathrm{~T}^{2 \mathrm{n}}$.

Hence;

$(\mathrm{T} / \mathrm{M}) * 2 \mathrm{~m}(\mathrm{~T} / \mathrm{M})^{2 \mathrm{n}}\{(\mathrm{T} / \mathrm{M}) * \mathrm{~m}(\mathrm{~T} / \mathrm{M})\}^{2}$

$=(\mathrm{T} / \mathrm{M}) * 2 \mathrm{~m}(\mathrm{~T} / \mathrm{M})^{2 \mathrm{n}}\{(\mathrm{T} / \mathrm{M}) * \mathrm{~m}(\mathrm{~T} / \mathrm{M}) \mathrm{n}\}^{2}$

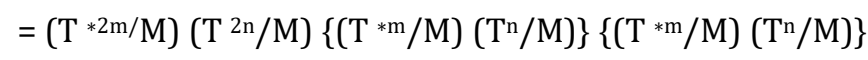

$=\left\{\left(\mathrm{T} * \mathrm{~m} \mathrm{~T}^{\mathrm{n}}\right)^{2} / \mathrm{M}\right\}\{\mathrm{T} * 2 \mathrm{~m} \mathrm{~T} 2 \mathrm{n} / \mathrm{M}\}$

$=\left\{(\mathrm{T} * \mathrm{~m} / \mathrm{M})\left(\mathrm{T}^{\mathrm{n}} / \mathrm{M}\right)\right\}^{2}(\mathrm{~T} / \mathrm{M}) * 2 \mathrm{~m}(\mathrm{~T} / \mathrm{M})^{2 \mathrm{n}}$

Thus $\mathrm{T} / \mathrm{M} \in(\mathrm{n}, \mathrm{mBQ})$.

\subsection{Theorem 2}

If $T \in B(H)$ is in $(n, m)$-power Class $(Q)$, then $T \in(n, m B Q)$.

Proof. If $\mathrm{T} \in(\mathrm{Q})$, then

$\mathrm{T} * 2 \mathrm{~m} \mathrm{~T}^{2 \mathrm{n}}=\left(\mathrm{T} * \mathrm{~m}^{\mathrm{n}}\right)^{2}$

Post multiplying both sides by $\mathrm{T} * 2 \mathrm{~m} \mathrm{~T} 2 \mathrm{n}$;

$\mathrm{T}^{* 2 \mathrm{~m}} \mathrm{~T}^{2 \mathrm{n}} \mathrm{T} * 2 \mathrm{~m} \mathrm{~T}^{2 \mathrm{n}}=\left(\mathrm{T} * \mathrm{~m} \mathrm{~T}^{\mathrm{n}}\right)^{2} \mathrm{~T} * 2 \mathrm{~m} \mathrm{~T}^{2 \mathrm{n}}$

$\mathrm{T} * 2 \mathrm{~m} \mathrm{~T}^{2 \mathrm{n}} \mathrm{T} * \mathrm{~m} \mathrm{~T}^{\mathrm{n}} \mathrm{T} * \mathrm{~m} \mathrm{~T}{ }^{\mathrm{n}}=\left(\mathrm{T} * \mathrm{~m} \mathrm{~T}^{\mathrm{n}}\right)^{2} \mathrm{~T} * 2 \mathrm{~m} \mathrm{~T}^{2 \mathrm{n}}$

$\mathrm{T} * 2 \mathrm{~m} \mathrm{~T}^{2 \mathrm{n}}(\mathrm{T} * \mathrm{~m} \mathrm{Tn})^{2}=\left(\mathrm{T} * \mathrm{~m} \mathrm{~T}^{\mathrm{n}}\right)^{2} \mathrm{~T} * 2 \mathrm{~m} \mathrm{~T}^{2 \mathrm{n}}$. 


\subsection{Theorem 3}

Let $S \in(n, m B Q)$ and $T \in(n, m B Q)$. If both $S$ and $T$ are doubly commuting, then

ST is in (n, mBQ).

Proof.

$(\mathrm{ST})^{* 2 \mathrm{~m}}(\mathrm{ST})^{2 \mathrm{n}}\left((\mathrm{ST}){ }^{* m}(\mathrm{ST})^{\mathrm{n}}\right)^{2}$

$=\mathrm{S}^{* 2 \mathrm{~m}} \mathrm{~T} * 2 \mathrm{~m} \mathrm{~S}^{2 \mathrm{n}} \mathrm{T}^{2 \mathrm{n}}\left((\mathrm{ST})^{* \mathrm{~m}}(\mathrm{ST})^{\mathrm{n}}\right)\left((\mathrm{ST}){ }^{* m}(\mathrm{ST})^{\mathrm{n}}\right)$

$=\mathrm{S}^{* 2 \mathrm{~m}} \mathrm{~T}^{* 2 \mathrm{~m}} \mathrm{~S}^{2 \mathrm{n}} \mathrm{T}^{2 \mathrm{n}}\left(\left(\mathrm{S}^{* \mathrm{~m}} \mathrm{~T} * \mathrm{~m}\right)\left(\mathrm{S}^{\mathrm{n}} \mathrm{T}^{\mathrm{n}}\right)\right)\left(\left(\mathrm{S}^{* \mathrm{~m}} \mathrm{~T}{ }^{* \mathrm{~m}}\right)\left(\mathrm{S}^{\mathrm{n}} \mathrm{T}^{\mathrm{n}}\right)\right)$

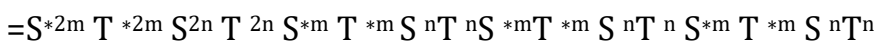

$=\mathrm{S}^{* 2} \mathrm{~T}^{* 2} \mathrm{~S}^{2} \mathrm{~T}^{2} \mathrm{~S}^{*} \mathrm{ST}{ }^{*} \mathrm{TS}^{*} \mathrm{ST} * \mathrm{~T}$

$=\mathrm{T} * 2 \mathrm{~m} T{ }^{2 n} \mathrm{~S}^{* 2 \mathrm{~m}} \mathrm{~S}^{2 \mathrm{n}} \mathrm{S}^{* \mathrm{~m}} \mathrm{~S}{ }^{\mathrm{n}} \mathrm{S}^{* \mathrm{~m}} \mathrm{~S}{ }^{\mathrm{n}} \mathrm{T} * \mathrm{~m} \mathrm{~T}{ }^{\mathrm{n}} \mathrm{T} * \mathrm{~m} \mathrm{~T}^{\mathrm{n}}$

$=T^{* 2 m} T^{2 n} S^{* 2 m} S^{2 n}\left(S^{* m} S^{n}\right){ }^{2} T * m T{ }^{n} T * m T^{n}$

$=T^{* 2 m} T^{2 n}\left(S^{* m} S^{n}\right)^{2} S^{* 2 m} S^{2 n} T * m+{ }^{n} T^{* m} T^{n}$ (Since $\left.S \in(n, m B Q)\right)$.

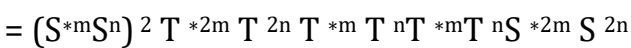

$=\left(S^{* m} S^{n}\right)^{2} T^{* 2 m} T^{2 n}\left(T * m T^{n}\right)^{2} S^{* 2 m} S^{2 n}$

$=\left(S^{* m} S^{n}\right)^{2}\left(T * m T^{n}\right)^{2} T * 2 m T^{2 n} S^{* 2 m} S^{2 n}($ Since $T \in(n, m B Q))$

$=\left(\left(\mathrm{S}^{* \mathrm{~m}} \mathrm{~S}^{\mathrm{n}}\right)(\mathrm{T} * \mathrm{mTn})\right)^{2} \mathrm{~T} *^{2} \mathrm{~m} \mathrm{~S} *^{2} \mathrm{~m} \mathrm{~T}^{2}{ }^{2} \mathrm{~S}^{2} \mathrm{~m}$

$=((\mathrm{S} * \mathrm{mT} * \mathrm{~m})(\mathrm{S} \mathrm{nTn}))^{2} \mathrm{~S} *^{2} \mathrm{~m} \mathrm{~T} *^{2} \mathrm{~m} \mathrm{~S}{ }^{2} \mathrm{n} \mathrm{T}{ }^{2} \mathrm{n}$

$=((\mathrm{ST}) * \mathrm{~m}(\mathrm{ST}) \mathrm{n})^{2}(\mathrm{ST}) *^{2} \mathrm{~m}(\mathrm{ST})^{2 \mathrm{n}}$

Thus $\mathrm{ST} \in($ n. $\mathrm{mBQ})$.

\subsection{Theorem 4}

Let $\mathrm{T} \in \mathrm{B}(\mathrm{H})$ be a class $(\mathrm{n}, \mathrm{mBQ})$ operator such that $\mathrm{T}=\mathrm{CT} * \mathrm{C}$ for positive integers $\mathrm{n}$ and $\mathrm{m}$ with $\mathrm{C}$ being a conjugation on $\mathrm{H}$. If $\mathrm{C}$ is such that it commutes with $\mathrm{T} *^{2} \mathrm{~m} \mathrm{~T}^{2} \mathrm{n}$ and $(\mathrm{T} * \mathrm{mTn})^{2}$, then $\mathrm{T}$ is an

$(\mathrm{n}, \mathrm{m})$-power class $(\mathrm{Q})$ operator.

Proof. Let $\mathrm{T} \in(\mathrm{n}, \mathrm{mBQ})$ and complex symmetric, then we have; $\mathrm{T} *^{2} \mathrm{~m} \mathrm{~T}{ }^{2} \mathrm{n}(\mathrm{T} * \mathrm{mTn})^{2}=(\mathrm{T} * \mathrm{mTn})^{2} \mathrm{~T} *^{2} \mathrm{~m} \mathrm{~T}^{2} \mathrm{n}$ And $\mathrm{T}=\mathrm{CT} * \mathrm{C}$.

Hence;

$\mathrm{T} *^{2} \mathrm{~m} \mathrm{~T}{ }^{2} \mathrm{n}(\mathrm{T} * \mathrm{mTn})^{2}=(\mathrm{T} * \mathrm{mTn})^{2} \mathrm{~T} *^{2} \mathrm{~m} \mathrm{~T}^{2} \mathrm{n}$

$\mathrm{T} *^{2} \mathrm{mT}{ }^{2} \mathrm{n}$ CT $\mathrm{nCCT} * \mathrm{~m} \mathrm{CCT} \mathrm{nCCT} * \mathrm{~m} \mathrm{C}=(\mathrm{T} * \mathrm{mTn})^{2} \mathrm{CT} \mathrm{nCCT} * \mathrm{~m} \mathrm{CCT} \mathrm{nCCT} * \mathrm{~m} \mathrm{C}$.

$\mathrm{T} *^{2} \mathrm{~m} \mathrm{~T}{ }^{2} \mathrm{n} \mathrm{CT} \mathrm{nT} * \mathrm{~m} \mathrm{~T} \mathrm{nT} * \mathrm{~m} \mathrm{C}=(\mathrm{T} * \mathrm{mTn})^{2} \mathrm{CT} \mathrm{nT} * \mathrm{mT} \mathrm{nT} * \mathrm{~m} \mathrm{C}$

$\mathrm{T} *^{2} \mathrm{~m} \mathrm{~T}{ }^{2} \mathrm{n} \mathrm{CT}{ }^{2} \mathrm{n} \mathrm{T} *^{2} \mathrm{~m} \mathrm{C}=(\mathrm{T} * \mathrm{mTn})^{2} \mathrm{CT} * \mathrm{mT} \mathrm{nT} * \mathrm{mT} \mathrm{nC}$ 
$\mathrm{T} *^{2} \mathrm{mT}^{2} \mathrm{n} \mathrm{CT} *^{2} \mathrm{~m} \mathrm{~T}{ }^{2} \mathrm{n} \mathrm{C}=(\mathrm{T} * \mathrm{mTn})^{2} \mathrm{C}(\mathrm{T} * \mathrm{mTn})^{2} \mathrm{C}$.

C commutes with $\mathrm{T} *^{2} \mathrm{~m} \mathrm{~T}{ }^{2} \mathrm{n}$ and $(\mathrm{T} * \mathrm{mTn})^{2}$ hence we obtain;

$\mathrm{T} *^{2} \mathrm{mT}^{2} \mathrm{nT} *^{2} \mathrm{mT}{ }^{2} \mathrm{n}=(\mathrm{T} * \mathrm{mTn})^{2}(\mathrm{~T} * \mathrm{mTn})^{2}$.

Which implies;

$\mathrm{T} *^{2} \mathrm{mT}{ }^{2} \mathrm{n}=(\mathrm{T} * \mathrm{mTn})^{2}$ and thus $\mathrm{T} \in(\mathrm{n}, \mathrm{m})$-power class $(\mathrm{Q})$.

\subsection{Theorem 5}

Let $T \in B(H)$ be $(n-1, m)$-class $(Q)$ operator, if $T$ is a complex symmetric

Operator such that $\mathrm{C}$ commutes with $(\mathrm{T} * \mathrm{mT})^{2}$ for a positive ineteger $\mathrm{m}$, then $\mathrm{T}$ is an $(\mathrm{n}, \mathrm{m})$-power class $(\mathrm{Q})$ operator.

Proof. With T being complex symmetric and (n-1, m)-class (Q), we have;

$\mathrm{T}=\mathrm{CT} * \mathrm{C}$ and $\mathrm{T} *^{2} \mathrm{mT}{ }^{2} \mathrm{n}-^{2}=(\mathrm{T} * \mathrm{mT} \mathrm{n}-1)^{2}$.

We obtain;

$\mathrm{T} *^{2} \mathrm{mT}{ }^{2} \mathrm{n}-{ }^{2} \mathrm{~T}^{2}=(\mathrm{T} * \mathrm{mT} \mathrm{n}-1)^{2} \mathrm{~T}^{2}$.

Hence;

$\mathrm{T} *^{2} \mathrm{~m} \mathrm{~T}{ }^{2} \mathrm{n}=(\mathrm{T} * \mathrm{mT} \mathrm{n}-1)^{2} \mathrm{~T} 2$.

$\mathrm{T} *^{2} \mathrm{~m} \mathrm{~T}{ }^{2} \mathrm{n}=\mathrm{T} *^{2} \mathrm{~m} \mathrm{~T}{ }^{2} \mathrm{n}-2 \mathrm{~T}^{2}=\mathrm{T}^{2} \mathrm{n}-{ }^{2} \mathrm{~T} *^{2} \mathrm{~m} \mathrm{~T}{ }^{2}$

$\mathrm{T} *^{2} \mathrm{~m} \mathrm{~T}^{2} \mathrm{n}=\mathrm{T}^{2} \mathrm{n}-^{2} \mathrm{~T} * \mathrm{mT} * \mathrm{mTT}=\mathrm{T}^{2} \mathrm{n}-^{2}$ CTCCTCCT $* \mathrm{mCCT} * \mathrm{mC}=\mathrm{T}^{2} \mathrm{n}-^{2}$ CTTT $* \mathrm{mT} * \mathrm{mC}$.

$=\mathrm{T} *^{2} \mathrm{~m} \mathrm{~T}{ }^{2} \mathrm{n}=\mathrm{T}^{2} \mathrm{n}-^{2} \mathrm{CT}{ }^{2} \mathrm{~T} *^{2} \mathrm{~m} \mathrm{C}=\mathrm{T}^{2} \mathrm{n}^{2} \mathrm{C}(\mathrm{T} * \mathrm{mT})^{2} \mathrm{C}$

Since $\mathrm{C}$ commutes with $(\mathrm{T} * \mathrm{mT})^{2}$ we obtain;

$\mathrm{T} *^{2} \mathrm{~m} \mathrm{~T}^{2} \mathrm{n}=\mathrm{T}^{2} \mathrm{n}-^{2}(\mathrm{~T} * \mathrm{mT})^{2} \mathrm{CC}=\mathrm{T}^{2} \mathrm{n}-2 \mathrm{~T} *^{2} \mathrm{~m} \mathrm{~T}{ }^{2} \mathrm{CC}=\mathrm{T}^{2} \mathrm{n}-{ }^{2} \mathrm{~T}^{2} \mathrm{~T} *^{2} \mathrm{~m} \mathrm{CC}=\mathrm{T} *^{2} \mathrm{~m} \mathrm{~T}{ }^{2} \mathrm{n}=(\mathrm{T} * \mathrm{mT} \mathrm{n})^{2}$

Hence $\mathrm{T}$ is n-power class (Q).

\section{Conclusion}

The study of class (n,mBD) operators will help in the enhancement of study of properties of various classes such as class (Q) operators, normal operators and binormal operators.

\section{Compliance with ethical standards}

\section{Acknowledgments}

The researchers appreciated all the comments and inputs made by experts before publication.

\section{Disclosure of conflict of interest}

The authors declared no conflict of interest. 


\section{References}

[1] Eiman H. Abood and Mustafa A. Al-loz. On some generalizations of (n,m)-normal powers operators on Hilbert space , Journal of progressive research in mathematics. 2016; 7(3): 2395 -0218.

[2] Jibril AAS. On Operators for which $\mathrm{T} * 2(\mathrm{~T}) 2=(\mathrm{T} * \mathrm{~T}) 2$, international mathematical forum. 5(46): 2255-2262.

[3] S Paramesh, D Hemalatha, VJ Nirmala. A study on n-power class (Q) operators, international research journal of engineering and technology. 2019; 6(1): 2395-0056.

[4] wanjala Victor, AM Nyongesa. On $(\alpha, \beta)$-class $(Q)$ Operators, international ournal of mathematics and its applications. 2021; 9(2): 111-113.

[5] Wanjala Victor, Beatrice Adhiambo Obiero., On almost class (Q) and class (M,n) operators ,international journal of mathematics and its applications. 2021; 9(2): 115-118.

[6] Wanjala Victor, Beatrice Adhiambo Obiero. on class (BQ) operators, Global Journal of advanced research. 2021; (4): 118-120.

[7] Wanjala Victor and Peter Kiptoo Rutto., K* Quasi-n- Class (Q) Operators, international journal of mathematics and its applications. 2021; 9(2): 189-193. 